\section{Prevalência e fatores associados à deficiência de ferro em lactentes atendidos em um centro de saúde-escola em Belém, Pará, Brasil}

\author{
Prevalence and factors associated with iron \\ deficiency in infants treated at a primary care \\ center in Belém, Pará, Brazil
}

Márcia Bitar Portella Neves 1 Edina Mariko Koga da Silva ${ }^{2}$ Mauro Batista de Morais 2

\footnotetext{
1 Departamento de Saúde Integrada, Universidade do Estado do Pará, Belém, Brasil.

2 Escola Paulista de Medicina, Universidade Federal de São Paulo, São Paulo, Brasil.

Correspondência M. B. Morais

Departamento de Pediatria, Escola Paulista de Medicina, Universidade Federal de São Paulo. Rua Pedro de Toledo 441, São Paulo, SP 04039-031, Brasil. mbmorais@osite.com.br
}

\begin{abstract}
The objective was to verify the occurrence of iron deficiency and associated factors in infants. This cross-sectional study included 365 infants (defined here as 6-24 months of age) treated at a primary care center in Belém, Pará, Brazil. Irondeficiency anemia (hemoglobin $<11 \mathrm{~g} / \mathrm{dl}$ and ferritin < $12 \mu \mathrm{g} / \mathrm{l}$ ) was diagnosed in $55.1 \%$ of the sample, depletion of body iron reserves (hemo-

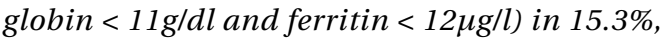
and iron sufficiency (hemoglobin $<11 \mathrm{~g} / \mathrm{dl}$ and ferritin $<12 \mu \mathrm{g} / \mathrm{l}$ ) in $18.1 \%$. The results of the logistic regression model showed associations be-

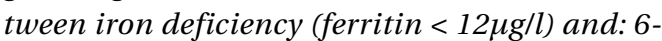
12 month age group, OR (odds ratio) $=3.67$ and 95\% CI: 1.93-7.04; non-utilization of iron-fortified formula as the first milk used after interrupting breastfeeding, $O R=1.93$ and 95\%CI: 1.04-3.60; and per capita income $\leq 1$ minimum wage, $O R=2.69$ and 95\%CI: 1.30-5.59. The occurrence of iron deficiency was high, showing the need to adopt effective measures to prevent this important public health problem.
\end{abstract}

Anemia; Iron Deficiency; Infant

\section{Introdução}

Estudos realizados em amostras da população infantil da Cidade de São Paulo, Brasil, mostram que a prevalência de anemia vem aumentando a partir da década de 1970 1. Outros estudos realizados em unidades básicas de saúde 2,3,4,5 também mostraram que a anemia ocorre com elevada prevalência em todas as regiões do Brasil onde foi investigada. A deficiência de ferro constitui a principal causa de anemia 6 e, na atualidade, é a deficiência nutricional com maior importância em saúde pública 7. Em Belém, Pará, Brasil, não existem informações sobre a ocorrência de anemia ferropriva em lactentes.

A concentração de hemoglobina sanguínea foi utilizada como indicador de anemia na maioria dos estudos realizados no Brasil 1,2,3,4,5, conforme recomendado pela Organização Mundial da Saúde (OMS) 6,7,8. No entanto, informações mais completas podem ser obtidas analisando-se outros indicadores do ferro corporal, especialmente a ferritina no soro, que indica a situação dos depósitos corporais de ferro ${ }^{9}$. Vale lembrar que os depósitos corporais de ferro são exauridos antes de ocorrer diminuição da concentração da hemoglobina, podendo ser caracterizada a depleção das reservas corporais de ferro sem anemia, etapa inicial da deficiência de ferro. Por sua elevada especificidade, a ferritina sérica permite, também, comprovar a etiologia ferropriva da anemia 7. 
Os lactentes constituem um grupo de risco para o desenvolvimento de deficiência de ferro 5,8. A possibilidade dos lactentes com deficiência de ferro não apresentarem pleno desenvolvimento neuropsicomotor, com alterações persistentes mesmo após a reversão do déficit do ferro corporal 10,11, além da ocorrência de outras manifestações negativas da deficiência de ferro, como diminuição do crescimento e maior susceptibilidade a infecções, que podem ocorrer nessa faixa etária, caracteriza esse grupo como uma das prioridades para a implementação de medidas para a prevenção desse grave distúrbio nutricional 12 .

Os objetivos deste estudo foram verificar a ocorrência de anemia ferropriva e de depleção das reservas corporais de ferro em lactentes com idade entre 6 e 24 meses e estudar os fatores associados com a deficiência de ferro.

\section{Metodologia}

Este estudo transversal foi realizado na Unidade Materno Infantil, Centro de Ciências Biológicas e da Saúde, que é vinculada à Universidade do Estado do Pará. A unidade está localizada no Distrito Administrativo do Bairro do Marco em Belém, com população total de 36.606 habitantes em 1999, estimando-se em 20,0\% (7.321) os menores de dois anos conforme a distribuição etária observada na cidade. Nessa unidade, são desenvolvidos todos os programas preconizados pelo Ministério da Saúde (MS) para a promoção de saúde da população pediátrica.

O cálculo do tamanho da amostra foi realizado com o programa Epi Info versão 6.04, utilizando a opção para estudos populacionais e descritivos. A proporção esperada de lactentes com anemia foi estimada em $60,0 \%$, conforme estudo piloto. Considerando a população de 7.321 menores de dois anos na região onde foi realizado o estudo e intervalo de confiança de 95,0\% (IC95\%) entre 55,0\% e 65,0\%, a amostra deveria ser constituída por 351 lactentes. Considerou-se a possibilidade de que $20,0 \%$ das crianças não preenchessem os critérios de inclusão ou suas mães não concordassem com a participação no estudo. Por tanto, estimou-se que 421 lactentes deveriam ser convidados para o estudo.

Durante a coleta de dados, foram convidados para participar na pesquisa todos os lactentes com idade entre 6 e 24 meses, atendidos no setor de vacinação da Unidade Materno Infantil, no horário entre 7 e 9 horas, durante o período no qual foi realizada a coleta de dados. Dos 410 lactentes que foram efetivamente con- siderados para serem incluídos no estudo, 6 $(1,5 \%)$ não foram incluídos por apresentarem evidência clínica de infecção aguda, 12 (2,9\%) por estarem recebendo medicamentos com ferro. As mães de outros 27 (6,7\%) lactentes não concordaram em participar da pesquisa. Assim, a casuística foi constituída por 365 lactentes.

Realizaram-se entrevistas com as mães dos lactentes para obtenção de informações referentes à renda per capita, antecedentes gestacionais e neonatais, alimentação atual e pregressa, incluindo duração do aleitamento natural. O questionário foi testado na pesquisa piloto que antecedeu a realização do estudo. A renda per capita foi dividida em duas categorias: menor ou igual a um salário mínimo ou maior do que um salário mínimo. Foram considerados prematuros os lactentes que nasceram com menos de 37 semanas de gestação, e baixo peso de nascimento foi caracterizado quando o mesmo foi inferior a $2.500 \mathrm{~g}$, de acordo com a classificação da OMS 13. Aleitamento natural exclusivo foi definido de acordo com a definição da OMS 14, sendo que os lactentes foram distribuídos em dois grupos: os que receberam aleitamento natural exclusivo até quatro meses e aqueles que receberam aleitamento natural exclusivo por mais de quatro meses. Indagou-se, também, se o lactente ainda recebia leite materno por ocasião do estudo e o primeiro tipo de leite artificial utilizado como complemento ou substituto do leite materno.

O peso foi obtido em balança mecânica para lactentes da marca Filizola, com capacidade máxima de $16 \mathrm{~kg}$, tarada e zerada.

A avaliação antropométrica para pesquisa de desnutrição energético-protéica foi realizada com o escore $\mathrm{Z}$ de peso para a idade, calculado no programa Epi Info 6.04 que considera os valores de referência do National Center for Health Statistics. Desnutrição energético-protéica foi caracterizada quando o escore $\mathrm{Z}$ do peso para a idade foi inferior a -2,0 desvios-padrão 15.

A coleta de sangue por punção venosa foi realizada no mesmo dia da entrevista. A concentração da hemoglobina foi determinada pelo método da cianometahemoglobina 16. A determinação da ferritina foi realizada pelo método da micropartícula enzimaimunoensaio (IM ${ }^{\circledR}$ Ferritina, ABBOTT). Todas as análises foram realizadas em duplicata, pelo Laboratório de Análises Clínicas Paulo Azevedo (Belém, Pará).

Anemia foi caracterizada quando a concentração de hemoglobina foi menor do que $11 \mathrm{~g} / \mathrm{dl}$, em concordância com o preconizado pela OMS para crianças com idade entre seis meses e sessenta meses 7,8. Depleção das reservas de ferro 
foi caracterizada quando o nível de ferritina sérica foi inferior a $12 \mathrm{mg} / \mathrm{l} 6$. Com base nos níveis da hemoglobina sanguínea e da ferritina no soro, os lactentes foram distribuídos em quatro categorias: (1) suficiência em ferro - valor de hemoglobina igual ou maior do que $11 \mathrm{~g} / \mathrm{dl}$ e nível de ferritina no soro igual ou maior do que $12 \mu \mathrm{g} / \mathrm{l}$; (2) anemia ferropriva - nível de hemoglobina menor do que $11 \mathrm{~g} /$ dl e nível de ferritina no soro menor do que $12 \mu \mathrm{g} / \mathrm{l}$; (3) depleção das reservas de ferro - valor de hemoglobina igual ou maior do que $11 \mathrm{~g} / \mathrm{dl}$ e nível de ferritina no soro inferior a $12 \mu \mathrm{g} / \mathrm{l}$ e (4) estado nutricional em ferro não classificável - valor de hemoglobina menor do que $11 \mathrm{~g} / \mathrm{dl}$ e nível de ferritina no soro igual ou maior do que $12 \mu \mathrm{g} / \mathrm{l}$. A denominação deficiência de ferro foi utilizada para o conjunto de lactentes com anemia ferropriva ou depleção das reservas de ferro, conforme as definições acima.

Constatada a deficiência de ferro nos lactentes, os responsáveis eram encaminhados para o ambulatório de pediatria para que o tratamento fosse realizado.

No dia do estudo, foi solicitada amostra de fezes para exame parasitológico. A pesquisa de parasitoses intestinais foi realizada pelos métodos de Faust e Bermann 17.

Para comparação das variáveis categóricas, utilizou-se o teste do qui-quadrado calculado com o emprego do programa Epi Info 6.04, adotando-se como nível de significância o valor de $5,0 \%(0,05)$. O modelo de regressão logística foi utilizado para análise multivariada tendo a deficiência de ferro como variável resposta definida com base nos níveis de ferritina inferiores a $12 \mathrm{mg} / \mathrm{l}$. Para a regressão logística, foi utilizado o programa MULTLR (Ludwig Institute for Cancer Research).

O projeto do estudo foi avaliado e aprovado pela Comissão de Ética da Escola Paulista de Medicina, Universidade Federal de São Paulo, de acordo com o cumprimento dos princípios éticos contidos na Declaração de Helsinki e da World Medical Association. No momento da inclusão no estudo, foi obtido consentimento informado por escrito dos responsáveis.

\section{Resultados}

Na Tabela 1, é apresentado o estado nutricional em ferro com base nos valores da hemoglobina e ferritina. Anemia ferropriva foi caracterizada em 55,1\%; depleção das reservas corporais de ferro, em 15,3\%; e suficiência em ferro, em $18,1 \%$ dos lactentes estudados. Portanto, a deficiência de ferro caracterizada por diminui-
Tabela 1

Estado nutricional em ferro, com base nos valores da hemoglobina e da ferritina sérica, em lactentes estudados em centro de saúde-escola em Belém, Pará, Brasil, 1999.

\begin{tabular}{|c|c|c|c|c|c|c|}
\hline \multirow{3}{*}{$\begin{array}{l}\text { Hemoglobina } \\
\text { (g/dl) }\end{array}$} & \multicolumn{4}{|c|}{ Ferritina $(\mu \mathrm{g} / \mathrm{l})$} & \multicolumn{2}{|c|}{ Total } \\
\hline & \multicolumn{2}{|c|}{$<12$} & \multicolumn{2}{|c|}{$\geq 12$} & \multirow[b]{2}{*}{$\mathrm{n}$} & \multirow[b]{2}{*}{$\%$} \\
\hline & $\mathrm{n}$ & $\%$ & $\mathrm{n}$ & $\%$ & & \\
\hline$<11,0$ & 201 * & 55,1 & $42^{\star \star}$ & 11,5 & 243 & 66,6 \\
\hline$\geq 11,0$ & $56^{\star \star \star}$ & 15,3 & 66\# & 18,1 & 122 & 33,4 \\
\hline Total & $257 \# \#$ & 70,4 & 108 & 29,6 & 365 & 100,0 \\
\hline
\end{tabular}

* Anemia ferropriva.

** Estado nutricional em ferro sem classificação.

*** Depleção das reservas corporais de ferro.

\# Suficiência em ferro.

\#\# Deficiência de ferro = (anemia ferropriva + depleção

das reservas corporais de ferro).

ção de ferritina no soro foi assinalada em 70,4\% dos lactentes.

Em 11,5\% dos lactentes, observou-se o valor da hemoglobina menor do que $11,0 \mathrm{~g} / \mathrm{dl}$ e nível de ferritina sérica maior ou igual a $12 \mathrm{mg} / \mathrm{l}$, não sendo possível caracterizar o estado nutricional em ferro, considerando que a depleção das reservas de ferro, aferida pela ferritina, deve preceder a diminuição do valor da hemoglobina. Esses lactentes foram excluídos das análises destinadas à avaliação dos fatores associados com a deficiência de ferro e foram encaminhados para avaliação diagnóstica e acompanhamento pediátrico. Portanto, a análise dos fatores associados com deficiência de ferro foi realizada com 323 lactentes (201 lactentes com anemia ferropriva, 56 com depleção das reservas de ferro sem anemia e 66 com suficiência em ferro).

A análise dos fatores associados com a deficiência de ferro nos lactentes estudados foi realizada, inicialmente, avaliando o grau de associação de cada variável isoladamente com o desfecho, ou seja, valor de ferritina no soro menor do que $12 \mu \mathrm{g} / \mathrm{l}$ (Tabela 2).

As variáveis analisadas estão divididas em duas categorias: antecedentes e no momento da realização do estudo. Em relação ao primeiro grupo, não foi identificada associação entre deficiência de ferro e as seguintes categorias: antecedentes de prematuridade e de baixo peso de nascimento, sexo e ausência de antecedente de aleitamento natural exclusivo por mais de quatro meses. Vale ressaltar que antecedente alimentar de aleitamento materno exclusivo por mais de quatro meses foi observado em 146 (45,2\%) dos 323 lactentes incluídos na análise dos fatores associados com deficiên- 
Análise bivariada dos fatores associados com deficiência de ferro (ferritina $<12 \mu \mathrm{g} / \mathrm{l}$ ) em lactentes $(\mathrm{n}=323)$ estudados em Belém, Pará, Brasil, 1999.

\begin{tabular}{|c|c|c|c|c|c|c|}
\hline \multirow[t]{3}{*}{ Fator } & \multicolumn{4}{|c|}{ Deficiência de ferro } & \multirow[t]{3}{*}{ Odds ratio (IC95\%) } & \multirow[t]{3}{*}{$\mathbf{p}$} \\
\hline & \multicolumn{2}{|c|}{$\operatorname{Sim}(n=257)$} & \multicolumn{2}{|c|}{ Não $(n=66)$} & & \\
\hline & $\mathrm{n}$ & $\%$ & $\mathrm{n}$ & $\%$ & & \\
\hline \multicolumn{7}{|l|}{ Antecedentes } \\
\hline Gemelaridade & 1 & 0,004 & 0 & 0,0 & Indefinido & 0,795 \\
\hline Prematuridade & 30 & 11,7 & 6 & 9,1 & $1,32(0,50-3,72)$ & 0,552 \\
\hline Peso ao nascer $<2.500 \mathrm{~g}$ & 16 & 6,2 & 4 & 6,1 & $1,03(0,31-3,79)$ & 0,960 \\
\hline Sexo masculino & 12 & 48,2 & 28 & 42,4 & $1,27(0,71-2,26)$ & 0,398 \\
\hline $\begin{array}{l}\text { Ausência de antecedente de aleitamento natural } \\
\text { exclusivo por mais de } 4 \text { meses }\end{array}$ & 117 & 45,5 & 29 & 43,9 & $1,07(0,60-1,90)$ & 0,817 \\
\hline $\begin{array}{l}\text { Ausência de uso de leite fortificado com ferro } \\
\text { como primeiro leite artificial }\end{array}$ & 174 & 67,7 & 35 & 53,0 & $2,29(1,24-4,21)$ & 0,004 \\
\hline \multicolumn{7}{|l|}{ Situação no momento do estudo } \\
\hline Faixa etária 6-12 meses & 147 & 57,2 & 21 & 31,8 & $2,86(1,56-5,30)$ & 0,0002 \\
\hline Renda per capita $\leq 1$ salário & 219 & 8,5 & 48 & 72,7 & $2,16(1,08-4,30)$ & 0,017 \\
\hline Não utilização de leite materno na época da avaliação & 130 & 50,6 & 46 & 69,7 & $0,45(0,24-0,82)$ & 0,0055 \\
\hline $\begin{array}{l}\text { Presença de desnutrição energético-protéica } \\
\text { (escore } Z<-2,0 \text { desvios-padrão de peso para a idade) }\end{array}$ & 11 & 4,3 & 5 & 7,6 & $0,55(0,17-1,88)$ & 0,336 \\
\hline Presença de parasitose intestinal* & 17 & 6,9 & 2 & 3,6 & $1,89(0,42-12,67)$ & 0,543 \\
\hline
\end{tabular}

* Exame parasitológico de fezes realizado em 247 crianças com deficiência de ferro e em 55 dos lactentes suficientes em ferro.

cia de ferro. Os lactentes que não receberam leite fortificado com ferro, como primeiro tipo leite artificial utilizado na alimentação, apresentaram chance (OR = 2,29; IC95\%: 1,24-4,21) mais elevada de serem portadores de deficiência de ferro (Tabela 2).

Com relação à situação das variáveis no momento do estudo, identificaram-se chances mais elevadas de deficiência de ferro entre as crianças com idade entre 6 e 12 meses (OR = 2,86; IC95\%: 1,56-5,30); entre aquelas cujas famílias referiram renda inferior a um salário mínimo (OR = 2,16; IC95\%: 1,08-4,30), e entre aquelas que faziam uso de leite materno no momento do estudo (OR = 0,45; IC95\%: 0,24-0,82). Não foi identificada associação entre deficiência de ferro com a presença de desnutrição energético-protéica ou parasitose intestinal (Tabela 2).

Na segunda etapa da avaliação estatística, foi realizada análise multivariada com o emprego de modelo de regressão logística (Tabela 3 ), incluindo as variáveis que isoladamente apresentaram associação estatisticamente significante com a deficiência de ferro. Confirmou-se que faixa etária, renda per capita e primeiro tipo de leite artificial utilizado pelo lactente apresentavam efetivamente relação com a ocorrência de deficiência de ferro. A única condição associada com deficiência de ferro na análise bivariada e que não manteve associação estatisticamente significante na análise multivariada foi a não utilização de leite materno no momento da avaliação.

\section{Discussão}

A elevada taxa de anemia (Tabela 1), encontrada no presente estudo, é compatível com pesquisas realizadas em outras regiões do país, caracterizando-a como um grave problema de saúde pública em lactentes de Belém, Pará, atendidos em um serviço público de saúde. Assim, em lactentes com idade entre 6 e 23 meses, estudados dentro da demanda espontânea em unidades básicas do Estado de São Paulo, observou-se anemia em taxas que variaram entre $47,8 \%$ e $68,7 \%$ dos lactentes, sendo a prevalência maior no interior do que na região metropolitana de São Paulo 5. Em outras regiões do Brasil, como Pernambuco 2, Porto Alegre 4, Rio de Janeiro 18 e em Criciúma 3, os estudos realizados revelaram anemia, respectivamente, em $82,8 \%, 65,6 \%, 54,0 \%$ e $78,3 \%$ das crianças estudadas.

Todos os estudos comentados no parágrafo anterior tiveram o diagnóstico de anemia es- 
Análise multivariada dos fatores associados com deficiência de ferro (ferritina $<12 \mu \mathrm{g} / \mathrm{l}$ )

em lactentes $(n=323$ ) de centro de saúde-escola em Belém, Pará, Brasil, 1999

\begin{tabular}{lcc}
\hline Variável & Odds ratio (IC95\%) & P \\
\hline Faixa etária 6-12 meses & $3,67(1,93-7,04)$ & 0,001 \\
Renda per capita $\leq$ um salário & $2,69(1,30-5,59)$ & 0,0077 \\
Não utilização de leite materno na época da avaliação & $0,81(0,42-1,54)$ & 0,5176 \\
Ausência de uso de leite fortificado com ferro como primeiro leite artificial & $1,93(1,04-3,60)$ & 0,0376 \\
\hline
\end{tabular}

tabelecido com base exclusivamente no valor da hemoglobina sanguínea, considerando-se o ponto de corte de $11,0 \mathrm{~g} / \mathrm{dl}$, conforme recomendado pela OMS 7,8. Em nosso estudo, para a classificação do estado nutricional em ferro, foi utilizada também a ferritina sérica como indicador dos depósitos corporais de ferro. A ferritina sérica é um bom indicador da deficiência de ferro, na ausência de infecção, pois apresenta alta especificidade na caracterização da anemia ferropriva, permitindo, ainda, caracterizar a depleção das reservas corporais de ferro 6,7,8,9.

Assim, deficiência de ferro (anemia ferropriva ou depleção das reservas corporais de ferro) foi caracterizada em 70,4\% dos 365 lactentes estudados. O IC95\% da taxa de deficiência de ferro situa-se entre $64,5 \%$ até $76,3 \%$.

Em nosso estudo, em 42 crianças, que correspondiam a $11,5 \%$ da amostra estudada, não foi possível definir o estado nutricional em ferro, uma vez que apresentavam diminuição dos níveis de hemoglobina e ferritina acima do limite adotado para caracterizar depleção das reservas corporais de ferro. Uma das explicações possíveis para esse achado seria a ocorrência de processos inflamatórios ou infecciosos virais nas semanas que antecederam a coleta de sangue, apesar de não terem sido detectadas evidências clínicas de infecção no momento do estudo. Nesse contexto, deve ser considerado que a ferritina pode elevar-se durante processos inflamatórios e infecciosos e assim permanecer por duas a quatro semanas após o período sintomático desses episódios 6,7,8,9. Sem a determinação dos níveis de ferritina, esse grupo de lactentes seria classificado como portadores de anemia provavelmente ferropriva, quando, na realidade, a diminuição dos níveis de hemoglobina poderia não estar relacionada com deficiência de ferro. Por essa razão, esses lactentes foram excluídos das análises dos fatores associados à deficiência de ferro.
Nossos resultados mostraram também que 15,3\% dos lactentes apresentavam depleção das reservas de ferro, mas ainda não apresentavam diminuição dos níveis de hemoglobina, ou seja, eram deficientes em ferro na sua fase pré-anêmica (depleção das reservas corporais de ferro). Esse aspecto foi avaliado por Hadler et al. 19, que encontraram depleção das reservas corporais de ferro em $20,0 \%$ dos lactentes estudados em unidade básica de saúde em Goiânia.

Os valores de ferritina, em nosso estudo, permitiram caracterizar deficiência de ferro em 70,4\% dos lactentes estudados. Desses lactentes, $55,1 \%$ apresentavam anemia ferropriva, e $15,3 \%$, depleção das reservas corporais de ferro, o que revela a gravidade desse déficit nutricional na população estudada.

Para análise dos fatores associados à deficiência de ferro, foram considerados os lactentes com suficiência em ferro que foram comparados com aqueles portadores de deficiência de ferro (anemia ferropriva ou depleção das reservas corporais de ferro). Não foram incluídos na análise os 42 lactentes que apresentaram diminuição da hemoglobina não acompanhada de diminuição da ferritina no soro.

Em nosso estudo, aleitamento materno exclusivo por mais de quatro meses não se caracterizou como fator de proteção contra deficiência de ferro entre os 6 e 24 meses. Estudos desenvolvidos na Cidade de São Paulo 20 e em Pernambuco 21 identificaram o aleitamento materno como protetor em lactentes no primeiro semestre de vida. Em outros estudos, como os realizados em centros de saúde de São Paulo 5 e em creches de Porto Alegre 4, não se identificou associação entre o aleitamento natural no primeiro semestre de vida e redução na prevalência de anemia no segundo semestre de vida. Em nosso estudo, a elevada freqüência de aleitamento natural exclusivo por mais de quatro meses mostra o provável efeito positivo das ações de incentivo ao aleitamento natural na 
Cidade de Belém, que foi identificada, em pesquisa realizada pelo MS em 2001 (Pesquisa de Prevalência do Aleitamento Materno nas Capitais e no Distrito Federal. Brasília: Fundo das Nações Unidas para a Infância; 2001), como uma das cidades com maior duração do aleitamento natural exclusivo no Brasil. No entanto, mesmo com a prática de aleitamento materno exclusivo por mais de quatro meses, a prevalência de anemia ferropriva e depleção das reservas corporais de ferro foi muito elevada tanto no segundo semestre como no segundo ano de vida, confirmando que, a partir dos seis meses de vida, outras fontes são necessárias para atender as necessidades de ferro 22 .

Na alimentação, observou-se, também, maior probabilidade de deficiência de ferro entre os lactentes que não receberam fórmula láctea modificada e fortificada com ferro como primeiro tipo de leite utilizado no processo de desmame.

Outro fator associado com deficiência de ferro foi a faixa etária entre os 6 e 12 meses, em relação ao segundo ano de vida. Essa associação pode ser explicada, teoricamente, pela maior velocidade de crescimento e menor freqüência e quantidade de consumo de alimentos fontes de ferro com alta biodisponibilidade 7 . Ou seja, crianças com idade entre 6 e 12 meses crescem mais, tomam mais leite de vaca não fortificado e comem menos carne que as crianças no segundo ano de vida. Esse aspecto foi observado em outro estudo realizado no Brasil 5.

Quanto à renda per capita, observou-se associação entre deficiência de ferro e renda per capita igual ou inferior a um salário mínimo, devendo ser assinalado que a deficiência de ferro foi caracterizada também em expressivo número nos lactentes pertencentes às famílias com renda superior a um salário mínimo. Associação entre anemia e menor renda também foi observada em outros estudos realizados no Brasil 1,20.

A alta prevalência de anemia por deficiência de ferro nos lactentes incluídos neste estudo, bem como na maioria dos estudos realizados em diferentes locais do país, confirma a necessidade de uma política de combate à deficiência de ferro, principalmente pela implementação de medidas preventivas. A OMS 12 recomenda a suplementação profilática de ferro para lactentes. Sigulem 20 , que vem estudando a deficiência de ferro no Brasil desde a década de 1970, recomendou a suplementação marcial profilática para lactentes 23 , em concordância com a proposta do Comitê de Nutrologia da Sociedade Brasileira de Pediatria. Esse aspecto contrasta com o fato de que, em nosso estudo, apenas 12 lactentes, dentre os 410 considerados, não foram incluídos no estudo por estarem recebendo suplementação de ferro ou ferroterapia oral. Assim, pode-se dizer que há a necessidade de efetivo engajamento das equipes de assistência à saúde no combate à deficiência de ferro, não somente prescrevendo a suplementação marcial, como também avaliando a adesão à prescrição e, ainda, adotando medidas efetivas para identificar lactentes com anemia ferropriva 24 . É provável que, pelo menos em parte, a baixa freqüência de prescrição de ferro para os lactentes da unidade básica de saúde, onde foi realizado este estudo, foi secundária à falsa impressão dos profissionais de saúde de que o aleitamento natural prolongado poderia proteger o lactente da anemia ferropriva.

Em conclusão, os resultados do presente estudo indicaram que a ocorrência de deficiência ferro é muito elevada nos lactentes com idade entre 6 e 24 meses, atendidos em uma unidade básica de saúde de Belém, vinculada a um serviço universitário, no qual 45,2\% dos lactentes tinham antecedente de aleitamento natural exclusivo por mais de quatro meses. A deficiência de ferro se associou com a não utilização de fórmula fortificada com ferro como primeiro tipo de leite utilizado na complementação ou substituição do leite materno, faixa etária entre 6 e 12 meses e renda inferior a um salário mínimo. Os resultados deste estudo indicam a necessidade da implementação de ações efetivas para a prevenção da deficiência de ferro que é um importante problema de saúde pública em lactentes. 


\section{Resumo}

Determinar a prevalência de deficiência de ferro e fatores associados em lactentes. Neste estudo transversal, foram estudados 365 lactentes atendidos em um centro de saúde-escola, em Belém, Pará, Brasil. Anemia ferropriva (hemoglobina $<11 \mathrm{~g} /$ dl e ferritina $<12 \mu \mathrm{g} / \mathrm{l}$ ) foi caracterizada em 55,1\% dos 365 lactentes; depleção das reservas corporais de ferro (hemoglobina $\geq 11 \mathrm{~g} / \mathrm{dl}$ e ferritina $<12 \mu \mathrm{g} / \mathrm{l}$ ), em 15,3\%; e suficiência em ferro (hemoglobina $\geq 11 \mathrm{~g} /$ dl e ferritina $\geq 12 \mu \mathrm{g} / \mathrm{l}$ ), em 18,1\%. Em 12,5\% dos lactentes, não incluídos na avaliação dos fatores associados com a deficiência de ferro, encontraram-se hemoglobina $<11,0 \mathrm{~g} / \mathrm{dl}$ e ferritina $\geq$ $12 \mu \mathrm{g} / \mathrm{l}$. Os resultados do modelo de regressão logística mostraram associação entre deficiência de ferro (ferri-

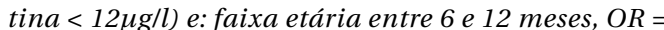
3,67 e IC95\%: 1,93-7,04; não utilização de fórmula fortificada com ferro como primeiro leite no desmame, $O R=1,93$ e IC95\%: 1,04-3,60; e renda per capita $\leq 1 \mathrm{sa}$ lário mínimo, $O R=2,69$ e IC95\%: 1,30-5,59. A prevalência de deficiência de ferro foi elevada, evidenciando a necessidade de implementação de ações efetivas para a prevenção desse importante problema de saúde pública.

Anemia; Deficiência de Ferro; Lactente

\section{Colaboradores}

Todos os autores participaram do planejamento do projeto, da interpretação e análise dos resultados e da redação e revisão do manuscrito. A coleta de dados foi efetuada sob a responsabilidade de M. B. P. Neves.

\section{Referências}

1. Monteiro CA, Szarfarc SC, Mondini L. Tendência secular da anemia na infância na cidade de São Paulo (1984-1996). Rev Saúde Pública 2000; 34 (6 Suppl):62-72.

2. Romani SAM, Lira PIC, Batista M, Siqueira LAS, Freitas CLC. Anemias em pré-escolares: diagnóstico, tratamento e avaliação. Arch Latinoam Nutr 1990; 41:159-67.

3. Neuman NA, Tanaka OT, Szarfarc SC, Guimarães PRV, Victora CG. Prevalência e fatores de risco para anemia no Sul do Brasil. Rev Saúde Pública 2000; 34:56-63.

4. Silva LSM, Giugliani ERJ, Aerts DRGC. Prevalência e determinantes de anemia em crianças de Porto Alegre, RS, Brasil. Rev Saúde Pública 2001; 35:66-73.

5. Torres MA, Sato K, Queiroz SS. Anemia em crianças menores de dois anos atendidas nas unidades básicas de saúde no Estado de São Paulo. Rev Saúde Pública 1994; 28:290-4.

6. Lanzkowsky P. Metabolismo do ferro e anemia por deficiência de ferro. In: Smith CH, Miller DR, Pearson HA, Baehner RL, McMillan CH, organizadores. Hematologia pediátrica. 4a Ed. Rio de Janeiro: Guanabara Koogan; 1982. p. 97-156.

7. United Nations Children's Fund. Preventing iron deficiency in women and children technical consensus on key issues. New York: International Nutrition Foundation; 1999.

8. DeMaeyer EM, Adiels-Tegman M. Prevalence of anaemia in the world. World Health Stat Q 1985; 38:302-16.

9. Rochina E, Diáz -de-Domingo NB, Lazarowski A. Ferritina sérica: su aplicación en clínica. Rev Biol Med Nuclear 1981; 13:29-35.

10. Pollitt E. Early iron deficiency anemia and later mental retardation. Am J Clin Nutr 1999; 69:115-9.

11. Lozoff B, De Andraca I, Castillo M, Smith JB, Walter T, Pino P. Behavioral and developmental effects of preventing iron-deficiency anemia in healthy full-term infants. Pediatrics 2003; 112: 846-54.

12. World Health Organization. Iron deficiency anaemia. Assessement, prevention and control. A guide for programme managers. Geneva: World Health Organization/The United Nations Children's Fund/United Nations University; 2001.

13. Anonymous. WHO: recommended definitions, terminology and format for statistical tables related to the perinatal period and use of a new certificate for cause perinatal deaths. Modifications recommended by FIGO as amended October 14, 1976. Acta Obstet Gynecol Scand 1977; 56:247-53.

14. World Health Organization. Indicators for assessing breastfeeding practices. Geneva: World Health Organization; 1991.

15. World Health Organization Expert Committee. Physical status: the use and interpretation of anthropometry. Geneva: World Health Organization; 1995. (WHO Technical Report Series 854).

16. Hailine A. Standard methods of clinical chemistry. New York: Academic Press; 1958.

17. Pessoa SB, Martins AV. Parasitologia médica. 11ạ Ed. Rio de Janeiro: Guanabara Koogan; 1988. 
18. Rodrigues CRM, Motta SS, Cordeiro AA, Lacerda EMA, Reichenhem ME. Prevalência e marcadores de risco associados em crianças entre 12 a 18 meses de idade atendidas nos ambulatórios do Instituto de Puericultura e Pediatria Martagão Gesteira. J Pediatr (Rio J) 1997; 73:189-94.

19. Hadler MCCM, Juliano Y, Sigulem DM. Anemia do lactente: etiologia e prevalência. J Pediatr (Rio J) 2002; 78:321-6.

20. Sigulem DM, Tudisco ES, Goldenberg P, Athaide MMM, Vaisman E. Anemia ferropriva em crianças do Município de São Paulo. Rev Saúde Pública 1978; 12:168-78.

21. Osório MM, Lira PIC, Batista-Filho M. Prevalence of anemia in children 6-59 months old in the state of Pernambuco, Brazil. Rev Panam Salud Pública 2001; 10:101-7.
22. Sichieri R, Szarfarc SC, Monteiro CA. Relação entre dieta e ocorrência de anemia ferropriva em crianças. J Pediatr (Rio J) 1988; 64:169-73.

23. Sigulem DM. Anemia nutricional ferropriva na primeira infância. In: Barbieri D, Palma D, organizadores. Gastroenterologia e nutrição. São Paulo: Atheneu; 2001. p. 297-310.

24. Morais MB, Sdepaninan VL. Sinais clínicos no diagnóstico de anemia: uma ação tardia e ineficiente no combate à deficiência de ferro. Rev Saúde Pública 2004; 38:329-30.

Recebido em 15/Set/2004

Versão final reapresentada em 25/Mai/2005 Aprovado em 07/Jun/2005 